\title{
EL PAPEL DE LA ACTIO TRIBUTORIA EN EL SISTEMA DE LOS PROCEDIMIENTOS CONCURSALES ROMANOS*
}

\author{
Alessandro Cassarino**
}

RESUMEN: La función de la actio tributoria era la de obtener la condena de aquel padre o amo cuyo sometido, hijo bajo potestad o esclavo, hubiese contraído obligaciones respecto de terceros, en el ejercicio de una negotiatio mediante una merx peculiaris, y después no las hubiese cumplido. En las Instituciones de Gayo 4.72 y las de Justiniano 4.7.3 se encuentra el análisis general de esta acción y la función de la merx peculiaris, como un patrimonio comercial autónomo. En caso de insolvencia del sometido, el pretor disponía que las mercancías remanentes y la recaudación peculiar fueran distribuidas entre todos los acreedores, comprendido el padre o amo, en partes proporcionales respectando el principio de la par condicio creditorum. Por la inexacta distribución debida al dolo, el pretor concedía la actio tributoria. La exposición de su régimen se completa con la lectura del título del Digesto justinianeo 14.4.1 y de la Paráfrasis griega a las Instituciones de Justiniano escrita por el jurista bizantino Teófilo.

PALABRAS CLAVE: Actio tributoria, merx peculiaris, par condicio creditorum, Paráfrasis griega de Teófilo.

\section{The Function of The ACTIO TRIBUtORIA IN ROMAN INSOLVENCY PROCEEDINGS}

ABSTRACT: The actio tributoria was granted by the Roman pretor to condemn a father or a master, whose son under authority (filius in potestate) or slave had contracted an obligation when he was trading with a merx peculiaris, and then he did not honor it. The general legal regime of this action is contained in Gaius' Institutes (4.72) and Justinian's Ins-

\footnotetext{
Traducción por Patricio Carvajal, Catedrático de Derecho Romano en la Universidad Católica de Santiago de Chile (CHILE). El texto, modificado y complementado, es una clase dictada en el magíster de Derecho de la Facultad de Ciencias Jurídicas de la Universidad Católica del Norte de Antofagasta, Chile, el 28 de noviembre de 2014.

Fecha de recepción: 29 de septiembre de 2015.

Fecha de aceptación: 13 de abril de 2016.

** Doctor en Derecho Romano y titular de una beca de investigación del Departamento de Derecho de la Universidad de Pisa (Italia). Correo electrónico: alexcass@email.it
} 
titutes (4.7.3), in which, it is also possible to find the regulation of the merx peculiaris as an independent fund. According to the Pretorian rules, in case of obligations not implemented by the son or slave, all the goods and money included in the merx peculiaris must be distributed pro rata among all the creditors who have proved their claim in an equal position (par condicio creditorum). The use of the actio tributoria was allowed only if the father or master made a fraudulent distribution. The knowledge of this actio is completed by the analysis of the title 14.1 of Justinian's Digest, and the Greek paraphrasis of Justinian's Institutes written by the Byzantine jurist Theophilus.

KEYWORDS: Actio tributoria - Merx peculiaris - Par condicio creditorum - Theophilus' Greek paraphrasis.

Sumario: 1) Introducción sobre el origen de las actiones adiecticiae qualitatis. 2) Las distintas actiones adiecticiae qualitatis. 3) El régimen de la actio tributoria en las Instituciones de Gayo y las de Justiniano. 4) La actio tributoria en el Digesto de Justiniano. 5) El dolo del potestad habiente en la distribución (tributio). 6) Breves notas introductorias a la Paráfrasis griega de Teófilo. 7) Análisis de su contenido. 8) Breves notas conclusivas. Bibliografía citada.

\section{1) INTRODUCCIÓN: EL ORIGEN DE LAS ACTIONES ADIECTICIAE QUALITATIS}

La presente contribución tiene por objeto el análisis, si bien solo en sus grandes líneas, de la actio tributoria, una de las seis actiones comúnmente designadas como actiones adiecticiae qualitatis (actio exercitoria, actio institoria, actio tributoria, actio quod iussu, actio de peculio et de in rem verso). Su función era la de obtener la condena de aquel padre o amo cuyo sometido, hijo bajo potestad o esclavo, hubiese contraído obligaciones respecto de terceros, en el ejercicio de una negotiatio, y después no las hubiese cumplido ${ }^{1}$. No obstante, para poder comprender su campo de aplicación es necesario esbozar preliminarmente un marco general.

En general véase, Buckland, William Warwick (1908) The Roman law of slavery. The condition of the slave in private law from Augustus to Justinian. Cambridge: at the University Press, p. 159, Burdese, Alberto (2010) Manuale di diritto privato romano. Rist. Milano: Wolters Kluwer Italia, p. 509, Cerami, Pietro (2010) "Introduzione allo studio del diritto commerciale romano". En Cerami, Pietro-Petrucci, Aldo: Diritto commerciale romano. Profilo storico. $3^{a}$ edición. Torino: Giappichelli Editore, p. 42, Guzmán Brito, Alejandro (2013) Derecho privado romano. $2^{\text {a }}$ edición. Santiago de Chile: Editorial Jurídica de Chile, II, p. 239, Lazo, Patricio (2015), "Acciones adyecticias y limitación de responsabilidad. Una hipótesis en torno a la justicia y la utilidad en el pensamiento de Ulpiano". Revista de Estudios Histórico-Jurídicos, vol. 37, pp. 1 y ss., Marrone, Matteo (2006) Istituzioni di diritto romano. Palermo: Palum- 
Todas estas acciones nacieron gracias a la intervención del pretor, que tuvo lugar al final de la Primera Guerra Púnica (264-241 a.C.), con el fin de superar la disparidad de tratamiento tanto en las adquisiciones realizadas por el hijo de familia o por el esclavo y transferidas directamente a favor del padre o amo, como en las deudas, por las cuales estos últimos no eran responsables ${ }^{2}$. Tal principio era propio del ius civile y se basaba en la simple consideración de que el esclavo, y el hijo bajo potestad, en cuanto sometidos al poder personal del padre, solo podían mejorar su situación económica, pero no empeorarla. Por el contrario, tal principio no valía para los eventuales delitos cometidos por los sometidos, caso en el cual el padre o amo tenía la elección entre pagar el daño o entregarlo a la persona ofendida mediante la noxae deditio ${ }^{3}$.

Si leemos, por ejemplo, las Instituciones de Gayo 1,52, se dice que: "Están bajo potestad de sus amos los esclavos. $Y$ dicha potestad es propia del derecho de gentes, pues observamos que es común a todas las gentes la potestad atribuida a los amos de decidir sobre la vida y la muerte de sus esclavos; y todo cuanto es adquirido por el esclavo pasa a ser propiedad del amo" ${ }^{4}$. Del mismo modo, aun en Gayo, 1,55 se afirma que: "Item in potestate nostra sunt liberi nostri quos iustis nuptiis procreavimus" ("También están bajo nuestra potestad los hijos que engendramos en justas nupcias").

El sistema del ius civile, entonces, no resultó adecuado para las nuevas exigencias de la economía romana entre los siglos III a.C. y III d.C., la cual experimentó un notable incremento de las relaciones de naturaleza comercial y, sobre todo, de la utilización de la mano de obra esclava ${ }^{5}$. En el período de tiempo al que nos hemos remontado aquí, en efecto, se verifica un gran desarrollo económico, cuyo centro neurálgico es Roma. Sobrevienen profundos cambios sociales, y el nacimiento de nuevas instituciones jurídicas es fruto de la intervención pretoria, de la que precisamente son ejemplos muy ilustrativos los cuatro contratos consensuales y las acciones adyecticias. Su cuna puede ser ubicada en la jurisdicción del pretor peregrino del 242 a.C., unida a la obra de la jurisprudencia

bo \& C. Editore, p. 195, Petrucci, Aldo (2014) Nozioni elementari di diritto romano. Pisa: Arnus University Books, p. 248, Serrao, Feliciano (2002) Impresa e responsabilità a Roma nell'età commerciale. Forme giuridiche di un'economia-mondo. Pisa: Pacini Editore, p. 24, TALAMANCA, Mario (1990) Istituzioni di diritto romano. Milano: Giuffrè, p. 84.

2 Cerami (2010) 42.

3 Para una amplia panorámica sobre el tema véase, en general, Serrao, Feliciano (2006) Diritto privato economia e società nella storia di Roma. Dalla società gentilizia alle origini dell'economia schiavistica. Napoli: Jovene Editore, I, p. 171.

4 "In potestate itaque sunt servi dominorum. Quae quidem potestas iuris gentium est; nam apud omnes peraeque gentes animadvertere possumus dominis in servos vitae necisque potestatem esse; et quodcumque per servum adquiritur, id domino adquiritur". Para el texto en latín y su traducción, seguimos el texto Samper, Francisco (2000) Instituciones de Gayo. Texto y Traducción. Santiago. Editorial Jurídica de Chile.

5 Cerami (2010) p. 27, Serrao (2002) 17. 
tardorrepublicana e imperial, verdadera maestra de finas interpretaciones creativas. La esclavitud aumentó de manera exponencial a causa de las conquistas imperialistas, proveyendo así las fuerzas de trabajo necesarias. Pero los esclavos también asumieron un papel fundamental como directores de empresas, favoreciendo el incremento y la difusión de las actividades comerciales, productivas y financieras. La actividad bancaria tuvo un relieve notable, de lo cual se encuentran testimonios precisos ya en las fuentes de finales del siglo III a.C., particularmente en las comedias de Plauto 6 . De hecho, este autor no requería a su público una específica preparación jurídico-económica para asistir a una comedia: por lo tanto, las tramas tratadas se incluían en el marco de un común conocimiento de los negocios de la vida cotidiana. Además, la referencia que hacen sus comedias a instituciones jurídicas de ámbito bancario ${ }^{7}$, aun cuando sus orígenes fueran griegos, permite entender el modo de actuar concreto de los banqueros en la sociedad de aquel tiempo.

Es en este momento, entonces, que interviene el pretor a través de una de las funciones que a él le correspondían, según lo describe un fragmento de Papiniano, extraído de su libro segundo de "Las Definiciones", D. 1, 1, 7, 1. En el fragmento se dice que el derecho pretorio es aquel que los pretores introdujeron adiuvandi vel supplendi vel corrigendi iuris civilis gratia propter utilitatem publicam, esto es, con el fin de ayudar, suplir y corregir el derecho civil por la utilidad pública. A partir de este fragmento, por lo tanto, se comprende por qué las actiones adiecticiae qualitatis nacieron al interior de la jurisdicción pretoria, la que persigue resolver la disparidad de tratamiento, antes señalada, a través de la aequitas ${ }^{8}$.

Además, también conviene precisar que el nombre actiones adiecticiae qualitatis proviene de la doctrina medieval, sobre la base de la interpretación de un fragmento del jurista Paulo, extraído de su libro vigésimo noveno del comentario al Edicto, recogido en D. 14, 1, 5, 19 , el cual sin

6 Andreau, Jean (1968) "Banque grecque et banque romaine dans le théâtre de Plaute et de Térence". Mélanges de l'École Française de Rome, vol. 80, p. 461, Petrucci, Aldo (1991) Mensam exercere. Studi sull'impresa finanziaria romana (II secolo a.C.-metà del III secolo d.C.). Napoli: Jovene Editore, 67.

7 Sobre las instituciones bancarias mencionadas en las comedias de Plauto, véase Petrucci (1991) 69 y ss.

8 Petrucci, Aldo (2012) Corso di diritto pubblico romano. Torino: Giappichelli Editore, p. 208.

$9 \quad$ "Item si servus meus navem exercebit et cum magistro eius contraxero, nibil obstabit, quo minus adversus magistrum experiar actione, quae mihi vel iure civili vel honorario competit: nam et cuivis alii non obstat hoc edictum, quo minus cum magistro agere possit: hoc enim edicto non transfertur actio, sed adicitur" ("Asimismo, si un esclavo mio explotare como naviero una nave, y yo hubiere contratado con el patrón de ella, nada obstará, para que yo ejercite contra el patrón la acción que me compete ó por derecho civil, ó por derecho honorario; porque tampoco obsta este Edicto á otro cualquiera, para que pueda ejercitar la acción contra el patrón; porque por este Edicto no se transfiere la acción, sino que se acumula"). Para el texto en latín, hemos seguido la versión de Momsen, Teodoro (1928) Corpus Iuris Civilis. Digesta. Belín: Wiedmanoss, 15a 
embargo corresponde solo a un caso específico, consistente en el incumplimiento de la obligación por parte del comandante de una nave en el giro de una empresa de navegación ${ }^{10}$.

Veamos rápidamente cuáles son tales acciones para pasar después al análisis más completo de la actio tributoria ${ }^{11}$.

\section{2) LAS DISTINTAS ACTIONES ADIECTICIAE QUALITATIS}

El orden que seguiré para la exposición de cada una de las actiones se conforma al modelo del Edicto perpetuo del pretor, redactado por el jurista Salvio Juliano durante el reinado del emperador Adriano (117-138 d.C.), el cual, según una parte de la doctrina ${ }^{12}$, puede considerarse como el orden cronológico de su creación: actio exercitoria, actio institoria, actio tributoria, actio quod iussu, actio de peculio et de in rem verso. Se trata de acciones diferentes, ya que también son diferentes los tipos de responsabilidad que recaen contra el padre del hijo bajo potestad o del amo del esclavo, como analizarenos en breve.

La actio exercitoria se funda en dos figuras particulares: el exercitor navis, el armador, y el magister navis, originalmente el esclavo o el hijo bajo potestad designado (praepositus) para la conducción de una nave en el ejercicio de una empresa de navegación con fines comerciales. Para comprender cuál es la responsabilidad que recae contra el armador (propietario o arrendatario de la nave), se debe observar cuáles son los límites de la llamada praepositio (designación), o sea, los poderes que este le otorgó al designado para el ejercicio de tal empresa. Por lo tanto, si se han respetado los límites de tal praepositio, el armador responderá por el total

edición, vol. I, p. 219. Para el texto en castellano, seguimos a García DEL Corral, Ildefonso Luis (2004) Cuerpo del Derecho Civil Romano. Valladolid: Lex Nova, reimpresión edición 1889 , t. I, p. 761

10 Talamanca (1990) 85.

11 Sobre esta acción, véanse Balestri Fumagalli, Marcella (1988). "L' "actio tributoria" nel sistema delle opere istituzionali di Gaio, di Giustiniano e di Teofilo". VV.AA. Atti del seminario sulla problematica contrattuale in diritto romano, Milano, 7-9 aprile 1987. Milano: Cisalpino-Goliardica, I, p. 122, Cerami (2010) 66, Chiusi, Tiziana Jolanda (1993). "Contributo allo studio dell'editto "De tributoria actione»". Memorie, Atti della Accademia Nazionale dei Lincei, Serie IX, vol. III, N4, p. 379, FöLDI, András (1996). "Remarks on the legal structure of enterprises in Roman Law". Revue Internationale de Droits de l'Antiquité, 43, p. 205, Guzmán Brito (2013) 239, Miceli, Maria (2001) Sulla struttura formulare delle 'actiones adiecticiae qualitatis'. Torino: Giappichelli Editore, p. 323, Petrucci, Aldo (2007) Per una storia della protezione dei contraenti con gli imprenditori. Torino: Giappichelli Editore, I, p. 79, Serrao (2002) p. 26, Petrucci, Aldo (2012). "Idee 'vecchie' e 'nuove' sulle attività imprenditoriali gestiti all'interno di un peculio". Bullettino dell'Istituto di Diritto Romano, 106, vol. 2, p. 295, SuÁrez Blázquez, Guillermo (2013). “Concurso mercantil de acreedores: vocatio in tributum. La "Acción tributoria” frente al dueño (empresario corrupto)". Revista de Derecho, Universidad del Norte, 39, p. 175, Valiño, Emilio (1967). "La 'actio tributoria". Studia et Documenta Historiae et Iuris, vol. 33, p. 103.

12 Cerami (2010) 44 contra De Ligt, Luuk (1999). "Legal history and economic history: the case of the actiones adiecticiae qualitatis". Tijdschrift voor Rechtsgeschiedenis, 67, p. 220. 
de la obligación (es, mutatis mutandis, como cuando en el derecho actual se habla del "poder suficiente" para contratar que puede tener, o no, un gerente de empresa; con la diferencia de que hoy existe al respecto una representación directa, mientras que en Roma esta no era en origen conocida) ${ }^{13}$.

El mismo discurso vale para la actio institoria, donde el titular de un establecimiento comercial designa (praeponit) previamente para su dirección a un esclavo o a un hijo, que en este caso toma el nombre de institor, para el ejercicio de una determinada actividad económica terrestre. Aquí el titular del establecimiento también responderá por el total de las deudas contraídas por el institor en el ejercicio de la empresa ${ }^{14}$. También en el caso del institor, todo gira alrededor del acto de la praepositio.

Por lo tanto, la importancia de las dos acciones y específicamente de las figuras del magister navis y del institor reside en el nacimiento por parte del pretor, de una primera forma de representación directa ${ }^{15}$.

La actio quod iussu, la actio de peculio y la actio de in rem verso (originalmente fundidas en una misma fórmula) formaban parte de una única cláusula edictal llamada edicto triple (triplex edictum) y todas implicaban la presencia de un peculio, como veremos a continuación.

La actio quod iussu se refería a una orden (iussum), que había sido dada por el padre o por el amo, a un tercero o al propio sometido, que tenía por fin la celebración de un determinado contrato entre dicho tercero y el hijo o esclavo. En este caso, habiendo mediado una orden directa del padre o amo, su tipo de responsabilidad es por el total de la obligación asumida, sin el beneficio de la limitación de esta al importe del valor del peculio ${ }^{16}$.

En cuanto a la actio de peculio, se refiere a una figura particular muy utilizada entre los romanos de la época: el peculio ${ }^{17}$. Este se puede considerar como un complejo de bienes atribuidos al propio hijo o esclavo para el ejercicio de una actividad. La particularidad reside en la circunstancia de que, teniendo el sometido la disposición material de los bienes, las eventuales adquisiciones que se pudieren realizar venían a incrementar el peculio mismo. La esencia de este tipo de acción se puede resumir en

13 Buckland (1908) 174, Cerami (2010) 56; 221, Földi, András (1995). "Die Entwicklung der sich auf die Schiffer beziehenden Terminologie im römischen Recht". Tijdschrift voor Rechtsgeschiedenis, 63, pp. 1 y ss., Guzmán Brito (2013) 224, Miceli (2001) 188, Serra (2002) 76.

14 Buckland (1908) 169, Cerami (2010) 42, Guzmán Brito (2013) 227, Miceli (2001) 188, Serrao, Feliciano (1971). "Institore (storia)". Enciclopedia del Diritto. Milano: Giuffrè Editore, vol. 21, p. 829, TALAMANCA (1990) 84.

15 Serrao (1971) 828.

16 Buckland (1908) 166, Cerami (2010) 42, Guzmán Brito (2013) 239, Miceli (2001) 309, Talamanca (1990) 84.

17 Buckland (1908) 187, Fleckner, Andreas Michael (2014). “The Peculium: A Legal Device for Donations to personae alieno iuri subiectae?". Gift Giving and the 'Embedded' Economy in the Ancient Worl, Heidelberg: Universitätsverlag Winter, p. 213. 
dos puntos fundamentales: en primer lugar, el titular de la potestad, en cuanto propietario formal del peculio, tenía la posibilidad de una satisfacción de los propios créditos sobre el hijo o el esclavo con preferencia respecto de todos los demás acreedores en el ámbito de la actividad ejercida en los límites del peculio. En segundo lugar, todos los demás acreedores eran satisfechos en sus créditos con el remanente que quedara del activo del peculio. Esto significa que quien reclamara su deuda primero entre los acreedores habría tenido una mayor posibilidad de ser satisfecho integralmente. Estamos hablando del principio prior tempore, potior iure ${ }^{18}$.

Posteriormente se agregó a la actio de peculio, la actio de in rem verso; una acción prevista para el caso de que el acreedor pida al padre o amo que responda también con los bienes adquiridos con el peculio y que han sido vertidos directamente en su propio patrimonio, es decir, aquellos que no fueron incorporados en el peculio mismo. He precisado poco antes que este tipo de actio originalmente se contenía en la actio de peculio, porque la fórmula dada por el pretor para ambas situaciones era única. A nivel procesal quiere decir que el pretor manda eventualmente a condenar en los límites del valor del peculio en caso de que exista, o bien, como ya se dijo, en el límite del enriquecimiento obtenido directamente por el padre o amo a partir de la gestión del peculio ${ }^{19}$.

La característica común de todas estas actiones que acabamos de analizar, concierne la condena por responsabilidad adjunta del titular de la potestad. Para dicha finalidad, en la intentio de la fórmula, en donde se incluía la pretensión del actor, era puesto el nombre de quien había celebrado el contrato - un esclavo o hijo bajo potestad o bien después de una cierta época, también un tercero hombre libre- y en la condemnatio, en donde se le otorgaba al juez el poder de condena o absolución, se insertaba el nombre del titular de la potestad.

\section{3) El RÉGIMEN DE LA ACTIO TRIBUTORIA EN LAS INSTITUCIONES DE GAYO Y LAS DE JUSTINIANO}

Hecha esta breve premisa sobre las cinco primeras actiones, pasemos ahora al análisis propiamente de la actio tributoria, una particular acción que prevé en su interior una responsabilidad para el padre o amo completamente diferente respecto de las precedentes, apenas antes vistas. Para comprender qué cosa se entiende por actio tributoria es necesario partir de la lectura de dos manuales de instituciones antiguos que nos han llegado directamente: las Instituciones de Gayo y las de Justiniano. Después examinaremos los puntos más salientes del título IV del libro decimo-

\footnotetext{
18 Cerami (2010) 42, Guzmán Brito (2013) 241, Miceli (2001) 229, Talamanca (1990) 84.

19 Buckland (1908) 176, Cerami (2010), Guzmán Brito (2013) 241, Miceli (2001) 294,
} 
cuarto del Digesto, dedicado precisamente a la actio tributoria, para terminar después, con un vistazo a la Paráfrasis de Teófilo.

Escribe Gayo, en sus Instituciones, en 4, 72: "Se estableció, además una acción tributoria contra el padre o amo, cuando el hijo o esclavo hacen un negocio con bienes de su peculio, sabiéndolo el padre o amo. Si se contrajera con ellos algo referente a este negocio, el pretor establece la regla de que todos los bienes del peculio afectos a este negocio y los ingresos procedentes de él deben distribuirse, en caso de deuda, entre el padre o amo y los otros acreedores en parte proporcional. $Y$ si los acreedores se quejan de que se les ha distribuido menos de lo que les correspondia, el pretor ofrece, para obtener lo que falta, esta acción llamada tributoria, esto es, de distribución" 20 .

Por su parte, reporta Justiniano en 4, 7, 3: "Introdujo también el pretor otra acción, que se llama tributoria. Porque si un esclavo negociara, sabiéndolo su señor, en mercaderías de su peculio, y con él se hubiere contratado alguna cosa por razón de tal comercio, el pretor falla de modo que todo lo que hubiere en estas mercaderias, y lo que por ellas se hubiere ganado, se distribuya a prorrata entre el señor, si algo se le debiere, y los demás acreedores. $Y$ como permite al mismo señor la distribución. $Y$ como permite al mismo señor la distribución, si alguno de los acreedores se querellase porque se le haya atribuido menos de lo que se hubiere debido, le concede esta acción, que se llama tributoria" 21 .

Ya en una primera lectura de los pasajes apenas citados, se nos hace notar cómo existe una gran similitud entre ambos, aunque en el texto de las Instituciones de Justiniano desaparece del todo la referencia al padre. Esto se debe al avance en materia de capacidad jurídica patrimonial de los hijos bajo potestad, como correctamente señalado por la doctrina predominante ${ }^{22}$, por el cual estos comenzaron a ser considerados responsables de las obligaciones que contrajeran en el ejercicio de una actividad comercial, lo cual probablemente se verificó hacia el I o II siglo d. C.

20 "Praetera tributoria quoque actio in patrem dominumve constituta est, cum filius servusve in peculiari merce sciente patre dominove negotietur. Nam si quid eius rei gratia cum eo contractum fuerit, ita praetor ius dicit, ut quidquid in his mercibus erit quod inde receptum erit, ita pater dominusve inter se, si quid debebitur, et ceteros creditores pro rata portione distribuant. Et si creditores querantur minus sibi distributum quam oporteret, in id quod deest hanc eis actionem pollicetur, quae, ut diximus, tributoria vocatur".

21 "Introduxit et aliam actionem praetor, quae tributoria vocatur. Namque si servus in peculiari merce sciente domino negotietur et quid cum eo eius rei causa contractum erit, ita praetor ius dicit, ut, quidquid in his mercibus erit quodque inde receptum erit, id inter dominum, si quid ei debebitur, et ceteros creditores pro rata portione distribuatur. Et quia ipsi domino distributionem permittit, si quis ex creditoribus queratur, quasi minus ei tributum sit, quam oportuerit, hanc ei actionem accommodat, quae tributoria appellatur".

22 En particular Petrucci (2007) 100. 
De esta evolución nos testimonia Ulpiano, en el libro sexagésimo tercero del comentario al Edicto, en D. 15, 1, 4423, donde se dice que si alguien había celebrado un contrato con un hijo bajo potestad, tiene a dos deudores: el hijo, por el total, y el padre dentro de los límites del peculio. Y también lo mismo comenta Paulo, en el libro sexagésimo primero del comentario al Edicto, en D. 15, 1, 4524, cuando observa que si el padre hubiera revocado al hijo su peculio, no por ello los acreedores no podrían accionar contra este último.

Si recorremos los textos, se nos hace evidente que al comienzo de ambos, en Gayo como algo sobrentendido y en Justiniano como algo explícito, se afirma que fue el pretor quien introdujo esta acción con el fin de que los acreedores pudieran contar con alguna tutela para la satisfacción de sus propios créditos. Tal tutela encuentra su razón en el conocimiento del padre, mencionado por Gayo, o del amo respecto de la actividad desarrollada por el hijo o por el esclavo con la merx peculiaris, o sea, con las mercancías del peculio. De esto resulta que al interior del peculio había algunas mercancías destinadas por el hijo o por el esclavo a actividades de naturaleza comercial. De hecho, la merx peculiaris, comúnmente es traducida por la doctrina como un patrimonio comercial autónomo ${ }^{25}$. Por lo tanto, en esta primera parte de la exposición institucional de Gayo y Justiniano se ha identificado tanto el elemento del conocimiento del padre o del amo, como el de la presencia, al interior del peculio, de la merx peculiaris; ambos necesarios para la aplicación de esta acción. A estos requisitos se ańade el de la insolvencia de la empresa comercial ejercitada por el sometido, la cual da ocasión a la apertura del procedimiento concursal.

El pretor dispone, entonces, que las mercancías remanentes y la recaudación peculiar sean distribuidas entre todos los acreedores, comprendido el padre o amo, en partes proporcionales, sin que estos últimos puedan satisfacerse en primer lugar. Se observará cómo hay aquí una total diferencia con la actio de peculio de la cual antes habíamos hablado, porque en ella al padre o al amo le era permitido satisfacerse previamente respecto de los acreedores ordinarios. En la actio tributoria, en cambio, esto no era posible. En efecto, para tal acción se habla de la par condicio creditorum, es decir, el igual tratamiento para todos los acreedores del hijo

\footnotetext{
23 "Si quis cum filio familias contraxerit, duos habet debitores, filium in solidum et patrem dumtaxat de peculio" " "Si alguno hubiere contratado con un hijo de familia, tiene dos deudores, al hijo por el todo, y al padre solamente respecto al peculio"). Véanse MoMmsen (1928) 233 y García DEL Corral (2004) 802.

24 "Ideoque si pater filio peculium ademisset, nibilo minus creditores cum filio agere possunt" (" $Y$, por lo tanto, si el padre hubiese quitado el peculio al hijo, pueden, sin embargo, los acreedores ejercitar su acción contra el hijo”). MOMMSEn (1928) 233 y GARCía DEL CoRRAL (2004) 802.

25 Para un estudio extenso sobre el concepto de merx peculiaris, véase Chiusi (1993) 283. Una afirmación explícita y directa se encuentra, por ejemplo, en Cerami (2010) 17.
} 
o del esclavo y no ya de una preferencia en relación al tiempo (prior tempore, potior iure) $)^{26}$.

Si después continuamos con nuestra lectura, se comprende como aquel que está llamado a la distribución es el padre o amo. Sin embargo, en el caso en que los acreedores se quejen de una inexacta distribución de las mercancías yacentes en el peculio o de las recaudaciones de dinero existentes en este, el pretor concederá la actio tributoria. En consecuencia, cuando el padre o el amo sean llamados a la distribución, hablamos de vocatio in tributum, $\mathrm{y}$, luego, si esta no es correcta, se pasará a la actio tributoria.

En verdad, no es claro en los dos textos -lo que ha despertado muchas dudas- si era el pretor quien establecía tal distribución, a consecuencia de la solicitud de los acreedores que acudían a él para obtener un decretum al respecto, o bien si, en cambio, bastaba que los acreedores mismos se apersonaran directamente al padre o amo, sin una previa adición del pretor. Sobre este punto de particular interés, Gayo habla de un genérico ita praetor ius dicit (así enuncia el derecho el pretor), sin ulteriores explicaciones. Esto ha hecho suponer que, entonces, al tiempo de Gayo no existía ningún acto preliminar del pretor, solicitado por uno o más acreedores, sino que se procedía directamente requiriendo la distribución al padre o amo.

Justiniano, en cambio, parece agregar un elemento más sobre este punto. Se afirma, en efecto, que el pretor permite la distribución al propio dueño (et quia ipsi domino distributionem permittit). El verbo permitto, por lo tanto, haría pensar en un acto de este magistrado -mencionado en el pasaje de las Instituciones por haber introducto dicha acción, y dispuesto como deban repartirse los bienes existentes y su precio- dirigido al amo para la distribución de las mercancías y de la correspondiente recaudación pecuniaria. Sobre este punto de extremo interés, intentaremos dar una respuesta en sede de las conclusiones pues nos queda por ver la Paráfrasis de Teófilo que podría venir en nuestra ayuda, con las oportunas cautelas, dada la distancia con las Instituciones de Gayo, aunque no con las de Justiniano, con las cuales es contemporánea.

\section{4) La ACtio tributoria EN El Digesto de Justiniano}

Concluido el discurso sobre las dos obras manualísticas, escritas y orientadas prevalentemente a los estudiantes, pasemos ahora a la lectura del régimen previsto en el libro 14, título IV, del Digesto que, por razones obvias de tiempo, solo será analizado en sus elementos más fundamentales. 
El título comienza con un fragmento de Ulpiano, extraído del libro vigésimo noveno de la obra de comentario al Edicto, donde, al elogiar el edicto, en el principium $^{27}$, se afirma que su utilidad no es mínima, porque mientras que para las otras obligaciones asumidas por el propio esclavo, en los límites del peculio, el amo puede deducir sus propios créditos antes que todos los demás, en el caso de que tuviera conocimiento (scierit) del ejercicio de una actividad empresarial con mercancías del peculio (merx peculiaris), él participará a la distribución de lo recaudado como si fuera un acreedor extraño. Aquí, por lo tanto, se verifica la particularidad de la llamada par condicio creditorum (igual posición de los acreedores), ya presente, como hemos visto, en los manuales de Gayo y de Justiniano. Después, Ulpiano prosigue en el parágrafo $1^{28}$, afirmando que, no obstante que el término "mercancía" deba considerarse restringida a tal punto que no quedarían comprendidas algunas categorías de actividades comerciales gestionadas por esclavos (tintorería, sastrería, textil y venta de los esclavos mismos), el jurista Pedio lo habría extendido a todo tipo de actividad empresarial. En el parágrafo $2^{29}$, se precisa que la merx peculiaris debe considerarse distinta del peculio mismo, porque, si este último se calcula deduciendo aquello que está debido al amo, esto no vale para la merx peculiaris, en cuanto el amo está en conocimiento de la actividad desarrollada por el esclavo. En el parágrafo $3^{30}$, Ulpiano explica que el conocimiento también comprende la voluntad y la tolerancia (patientiam),

27 D. 14, 4, 1 pr.: "Huius quoque edicti non minima utilitas est, ut dominus, qui alioquin in servi contractibus privilegium habet (quipe cum de peculio dumtaxat teneatur, cuius peculii aestimatio deducto quod domino debetur fit), tamen, si scierit servum peculiari merce negotiari, velut extraneus creditor ex hoc edicto in tributum vocatur" ("Tampoco es pequeña la utilidad de este Edicto, para que el señor, que de otro modo tiene privilegio en los contratos de un esclavo, porque cuando se obliga solamente por el peculio, se hace la estimación de este peculio deducido lo que se debe al señor, sea, no obstante, llamado á contribución por este Edicto, como acreedor extraño, si hubiere sabido que el esclavo negocia con mercancía del peculio"). Véanse MOMMSEN (1928) 223 y García del Corral (2004) 773.

28 D. 14, 4, 1, 1: "Licet mercis appellatio angustior sit, ut neque ad servus fulones vel sarcinatores, vel textores, vel venaliciarios pertineat, tamen Pedius libro quinto decimo scribit, ad omnes negotiationes porrigendum Edictum" ("Aunque la denominación de mercancía sea tan restricta, que no corresponda ni á los essclavos bataneros, ni á los sastres, ni á los tejedores, ni á los revendedores, sin embargo, escribe Pedio en el libro décimo quinto, que se ha de extender el Edicto á todas las negociaciones"). Véanse Mommsen (1928) 223 y García del Corral (2004) 773.

29 D. I 4, 4, I, 2: "Peculiarem autem mercem non sic uti peculium accipimus, quippe peculium deducto quod domino debetur accipitur, merx peculiaris etiamsi nibil sit in peculio, dominum tributoria obligat, ita demum si sciente eo negotiabitur" ("Más la mercancía del peculio no la consideramos como peculio, porque peculio se entiende deducido lo que se debe al señor, y la mercancía del peculio, aunque nada haya en el peculio, obliga al señor por la acción tributoria, pero solamente si se negociare sabiéndolo él'). Véanse MOMMSEn (1928) 223 y García del CoRRAL (2004) 773.

30 D. 14, 4, 1, 3: "Scientiam hic eam accipimus, quae habet et voluntatem, sed ut ego puto, non voluntatem, sed patientiam: non enim velle debet dominus, sed non nolle. Si igitur scit et non protestatur et contra dicit, tenebitur actione tributoria" ("Tomamos aqui por conocimiento, el que tiene también voluntad; pero según opino, no voluntad, sino tolerancia, porque el señor debe 
porque, para ser responsable, no es necesario que el amo quiera explícitamente, puesto que solo deja de ser responsable aquel que no quiere. Esto implicaría, para expresarlo en otras palabras, que no se oponga o declare estar en contra de la actividad comercial.

Como se ve, podemos aventurar que en cada uno de los parágrafos del fragmento 1, Ulpiano reporta consideraciones generales sobre los requisitos para intentar la actio tributoria ${ }^{31}$.

Tomemos ahora en consideración el fragmento 5 (D. 14, 4, 5), uno de los más largos dentro del título sobre la actio tributoria, que describe toda una serie de casos prácticos respecto de quien tiene el derecho de participar en la repartición de la merx peculiaris. En tal fragmento, querría poner la atención en dos puntos. El primero es el parágrafo $15^{32}$, cuando se trata de una pluralidad de acreedores, pero de patrimonios comerciales separados gestionados por un mismo esclavo. El caso contemplado prevé algunos acreedores de una actividad de comercio de sayos y otros correspondientes a otra actividad comercial que tenía por objeto lienzos. Ulpiano reputa que los acreedores de ambas deben ser considerados separadamente, porque son tales no respecto del esclavo, sino que respecto del patrimonio comercial. Tal criterio después es repetido en la continuación del texto, en el parágrafo $16^{33}$, donde los acreedores de una misma actividad desarrollada en dos locales comerciales diferentes, no pueden considerarse en su conjunto: los del local puesto en Hucino (un lugar de Roma antigua) son distintos de los de aquel situado al otro lado del Tíber. La razón reside en evitar que algunos sean satisfechos con las cosas y con el patrimonio comercial del otro local. Por lo tanto, en líneas generales,

no querer, sino no oponerse; así, pues, si lo sabe, y no protesta, y contradice, se obligará por la acción tributoria”). Véanse Mommsen (1928) 223 y García del Corral (2004) 773.

31 Miceli (2001) 325.

32 D. 14, 4, 5, 15: "Si plures habuit servus creditores, sed quosdam in mercibus certis, an omnes in isdem confundendi erunt et omnes in tributum vocandi? Ut puta duas negotiationes exercebat, puta sagariam et linteariam, et separatos habuit creditores. Puto separatim eos in tributum vocari: unusquisque enim eorum merci magis quam ipsi credidit" ("Si el esclavo tuvo muchos acreedores, pero algunos por ciertas mercancias, ¿se habrán de confundir todos en las mismas, y habrán de ser llamados todos a la contribución, por ejemplo, si ejercía dos negociaciones, como la de sayos y la de lienzos, y tuvo acreedores separados? Opino, que se les llama separadamente a la contribución, porque cada uno de ellos prestó más hien a la mercancía, que al mismo esclavo"). Véanse Mommsen (1928) 224 y García del Corral (2004) 775.

33 "Sed si duas tabernas eiusdem negotiationis exercuit et ego fui tabernae verbi gratia quam ad Bucinum habuit ratiocinator, alius eius quam trans Tiberim, aequissimum puto separatim tributionem faciendam, ne ex alterius re merceve alii indemnes fiant, alii damnum sentiant" ("Pero si tuvo dos tiendas de la misma negociación, y yo fui contador, por ejemplo, de la tienda que tuvo en Hucino, y otro de la que tuvo al otro lado del Tiber, considero muy justo que se haya de hacer por separado la contribución, para que unos no sean indemnizados con cosa ó mercancía de otro, y otros experimenten daño"). Véanse Mommsen (1928) 224 y García del Corral (2004) 775 . 
podemos afirmar que a un distinto patrimonio comercial corresponde una distinta distribución.

Otro punto, incluido en el parágrafo $19^{34}$, se refiere al caso en que se presente un solo acreedor el cual sea íntegralmente satisfecho. En tal situación, dice Ulpiano, pues se trata de un procedimiento concursal y la regla es que la distribución debe ser efectuada en proporción a cuanto le sea debido a cada uno, el acreedor deberá prestar una estipulación de garantía, de modo que, si se presentan otros acreedores, estos puedan ser satisfechos en proporción a sus créditos.

\section{5) EL DOLO DEL POTESTAD HABIENTE EN LA DISTRIBUCIÓN (TRIBUTIO)}

Llegados a este punto, la pregunta fundamental es entender si existe algún elemento esencial que permita explicar la injusta distribución que conduce a la aplicación de la actio tributoria. De la lectura del título que estamos analizando, la respuesta, lo anuncio de inmediato, debe centrarse en el dolo de aquel que realiza la distribución misma ${ }^{35}$. En base al supuesto que fuera dicho elemento el dominante en la actio tributoria, parte de la doctrina ${ }^{36}$ sostiene que ella no puede incluirse entre las actiones adiecticiae qualitatis porque representaba una sanción para la distribución dolosa y no para afirmar una responsabilidad adjunta del titular de la potestad. Sin embargo, me convence mucho más otra lectura interpretativa $^{37}$, que la comprende entre las actiones adiecticiae, por la simple consideración que también la actio tributoria presupone un contrahere (la celebración de contratos) por parte de un sometido que encuentra su ratio en el conocimiento (scientia) del titular de la potestad ${ }^{38}$.

34 "Tributio autem fit pro rata eius quod cuique debeatur. Et ideo, si unus creditor veniat desiderans tribui, integram portionem consequitur: sed quoniam fieri potest, ut alius quoque vel alii existere possint mercis peculiaris creditores, cavere debet creditor iste pro rata se refusurum, si forte alii emerserint creditores" ("Mas la contribución se hace á prorrata de lo que á cada cual se debe; y por esto, si se presentara un solo acreedor pretendiendo que se le entregue integra la porción, lo consigue. Pero como puede suceder, que puedan existir también otro, ú otros acreedores de la mercancía del peculio, debe este acreedor dar caución de que él les reembolsará á prorrata, si acaso salieren otros acreedores"). Véanse MoMmsen (1928) 224 y GARCía del Corral (2004) 776.

35 En este sentido, Chiusi (1993) 363, Guzmán Brito (2013) 249, VAliño (1967) 116.

36 VALIÑo (1967) 123.

37 Cerami (2010) 43.

38 Lenel, Otto (1927). Das Edictum Perpetuum. Leipzig: Verlag von Bernhard Tauchnitz, 271 pp., describe el contenido del edicto sobre la actio tributoria, con estas palabras: "Qui merce peculiari sciente eo, in cuius potestate erit, negotiabitur, si quid cum eo eius mercis nomine contractum erit, quod ex ea merce erit eoue nomine receptum erit, eum, in cuius potestate erit, si quid ei debebitur, cum creditoribus mercis pro rata eius quod cuique debebitur in tributum vocabo" ("Quien gestionará una actividad comercial con mercancías del peculio sabiéndolo el potestad habiente, si se contratará algo con él en razón de aquellas mercancías del peculio, o se 
Ulpiano habla del dolo en la distribución, siempre en el libro vigésimo noveno del comentario al Edicto, en D., 14, 4, 3, 139, con referencia a las situaciones del esclavo del pupilo o del demente que ejerza una actividad empresarial con el conocimiento del tutor o curador. Otros ejemplos de comportamientos sobre los cuales se puede identificar el dolo de quien efectúa la distribución de los montos debidos a los acreedores, son reportados siempre por el mismo jurista en D., 14, 4, 7, $2^{40}$. Aquí se considera el comportamiento de quien había distribuido menos de lo que se debía y de quien no había distribuido nada, porque, escribe Ulpiano, es comparable con quien había distribuido menos. Existe una exención solo en el caso de que se haya hecho una errónea distribución, por menos de lo debido, cuando el encargado de ella no haya conocido el activo poseído por el esclavo en la merx peculiaris. Sin embargo, por el otro lado, si se descubre que se ha repartido menos y el encargado de ello no procede a efectuar una nueva distribución, le será imputado el dolo. Continúa en el parágrafo $3^{41}$ Ulpiano, afirmando que actúa con dolo quien dejó perecer las cosas al interior de la merx peculiaris, sin evitarlo, o también quien haya revocado la merx o vendido en menos las cosas y no haya recuperado de los compradores al menos el precio. Así, en el parágrafo $4^{42}$ según la

recibirá algo de aquella mercancía o en razón de ella, llamaré a la distribución el potestad habiente, si algo se le hubiese debido, junto con los acreedores de la mercancía proporcionalmente a prorrata"). Para una hipótesis reconstructiva de la fórmula de la actio tributoria véase MANTovani, Dario (1999). Le formule del processo privato romano. Per la didattica delle Istituzioni di diritto romano. Padova: Milani, p. 80.

39 "Si servus pupilli vel furiosi sciente tutore vel curatore in merce peculiari negotietur..." "Si el esclavo del pupilo, ó del furioso, negociara con mercancía del peculio sabiéndolo el tutor ó el curador... "). Véanse Mommsen (1928) 223 y García del Corral (2004) 773.

40 "Si cuius dolo malo factum est, quo minus ita tribueretur, in eum tributoria datur, ut quanto minus tributum sit quam debuerit, praestet: quae actio dolum malum coercet domini. Minus autem tribuere videtur etiam si nihil tributum sit. Si tamen ignorans in merce servum habere minus tribuit, non videtur dolo minus tribuisse, sed re comperta si non tribuat, dolo nunc non caret. Proinde si sibi ex ea merce solvi fecit, utique dolo videtur minus tribuisse" " $S i$ por dolo malo de alguno sucedió, que asi no se hiciese la contribución, se da contra él la acción tributoria, para que entregue cuanto se haya contribuido de menos, que lo que hubiere debido; cuya acción corrige el dolo malo del señor. Pero se entiende que contribuye con menos, aunque no se haya contribuido con nada. Pero si ignorando que tiene el esclavo en el comercio, contribuyó con menos, no se entiende que dolosamente contribuyó con menos; mas si sabido el caso no contribuyera, no queda entonces exento de dolo; por consiguiente, si hizo que de aquella mercancía se le pagara á él, se entiende, á la verdad, que dolosamente contribuyó con menos"). Véanse MoMMSEN (1928) 223 y García del Corral (2004) 776.

41 D. I 4, 4, 7, 3: "Sed et si mercem perire passus est aut eam avertit aut vilioris data opera distraxit vel si ab emptoribus pretium non exegerit, dicendum erit teneri eum tributoria, si dolus intervenit ("Pero también si consintió que se perdiese la mercancía, ó la quitó, ó la vendió de intento por menos, ó si no hubiere cobrado de los compradores el precio, se habrá de decir, que queda él obligado por la acción tributoria, si medió dolo"). Véanse MOMMSEN (1928) 223 y García del Corral (2004) 776.

42 D. 14, 4, 7, 4: "Sed et si negaverit dominus cuiquam deberi, videndum erit, an tributoriae locus sit. Et est verior Labeonis sententia tributoria locum habere: alioquin expediet domino negare" ("Pero aunque hubiere negado el señor que se debia a alguno, se habrá de ver, si habrá 
opinión de Labeón, incurre en dolo el amo que haya negado la existencia de los créditos de terceros.

También se prevé la posibilidad de que el amo se niegue a proceder a la distribución. En este caso, afirma Pedio, quien es mencionado por Ulpiano en el parágrafo $1^{43}$, si el amo cede el peculio y la merx peculiaris, debe ser excluido y la distribución será efectuada normalmente por un juez designado por el pretor.

La actio tributoria, escribe Ulpiano en el parágrafo $5^{44}$, será otorgada sin límites de tiempo, por lo tanto, también contra el heredero del amo del esclavo, pero limitadamente en cuanto a lo que haya recibido como enriquecimiento.

Como ha sido puesto de relieve por un sector de la doctrina ${ }^{45}$ que me parece convincente, en el estado actual de nuestro conocimiento, la actio tributoria tendría naturaleza solo reipersecutoria y no penal. Esto sería demostrable por las palabras de Ulpiano, en el vigésimo noveno libro de su comentario al Edicto, en D., 14, 4, 9, $1^{46}$ y de Gayo, en el libro noveno de su comentario al edicto provincial, en D., 14, 4, $11^{47}$, donde se aclara que la elección entre la aplicación de la actio de peculio y de la

lugar a la acción tributoria. Y más verdadera la opinión de Labeón, de que tiene lugar la acción tributoria; pues de otro modo al señor le convendrá negar"). Véanse MOMMSEN (1928) 223 y García del Corral (2004) 776.

43 D. I 4, 4, 7, I: "Quid tamen si dominus tribuere nolit nec hanc molestiam suscipere, sed peculio vel mercibus cedere paratus sit? Pedius refert audiendum eum, quae sententia habet aequitatem: et plerumque arbitrum in hanc rem praetor debebit dare, cuius interventu tribuantur merces peculiares" ("¿Pero qué se dirá, si el señor no quisiera contribuir, ni tomarse esta molestia, pero estuviera dispuesto á hacer cesión del peculio, ó de las mercancias? Dice Pedio, que ha de ser oido; cuya opinión es equitativa. Y las más de las veces deberá nombrar el Pretor para este caso un árbitro, con cuya intervención se contribuya con las mercancías del peculio"). Véanse Mommsen (1928) 224 y García del Corral (2004) 776.

44 D. 14, 4, 7, 5: "Haec actio et perpetuo et in heredem datur de eo dumtaxat quod ad eum pervenit" ("Esta acción se da tanto perpétuamente, como contra el heredero, solamente por aquello que fue á poder de este”). Véanse Mommsen (1928) 224 y García del Corral (2004) 776.

45 MiCELI (2001) 330.

46 "Eligere quis debet, qua actione experiatur, utrum de peculio an tributoria, cum scit sibi regressum ad aliam non futurum. Plane si quis velit ex alia causa tributoria agere, ex alia causa de peculio, audiendus erit" ("Cualquiera debe elegir la acción de que usará, si la de peculio, ó la tributoria, sabiendo que no habrá de tener regreso para la otra. Mas si alguien quisiera ejercitar por una causa la acción tributoria, y por otra la de peculio, deberá ser oido"). Véanse MoMMSEN (1928) 224 y García del Corral (2004) 777.

47 "Aliquando etiam agentibus expedit potius de peculio agere quam tributoria: nam in hac actione de qua loquimur hoc solum in divisionem venit, quod in mercibus est quibus negotiatur quodque eo nomine receptum est: at in actione de peculio totius peculii quantitas spectatur, in quo et merces continentur" ("Algunas veces también conviene más á los actores ejercitar la acción de peculio, que la tributoria; porque en esta acción, de que hablamos, se comprende en la división solamente lo que hay en las mercancias, con que se negocia, y lo que con motivo de ellas se percibió. Mas en la acción de peculio se atiende á la cuantía de todo de peculio, en el cual se comprenden también las mercancías”). Véanse Mommsen (1928) 224 y García del Corral (2004) 777. 
actio tributoria es alternativa. Justamente, si la actio tributoria fuese de naturaleza penal, la elección entre la una y la otra no habría tenido sentido.

\section{6) Breves notas introductorias a la Paráfrasis griega de TEÓFILO}

Finalmente, llegamos a Teófilo, profesor de Constantinopla, el cual tuvo un papel activo en la Compilación de Justiniano como miembro de las comisiones que redactaron el primer Código del año 529 d.C. y el Digesto, el 533 d.C.; y, finalmente, en el mismo año, junto a Doroteo, profesor de Beirut, y a Triboniano, escribió las Instituciones. Agréguese que algunos autores están persuadidos de que Teófilo mismo hubiera muerto poco después de la publicación del Digesto y de las Instituciones, porque al inicio del 534, cuando se inciaron los trabajos del segundo Código, su nombre desaparece ${ }^{48}$.

A él se atribuye habitualmente una Paráfrasis griega de las Instituciones de Justiniano. "Paráfrasis" viene del término griego paraphrázō que significa "decir con otras palabras" y, para ser más precisos, no es otra cosa que una exposición de un texto con palabras apropiadas y la adición de aclaraciones.

El problema que se presentó a la época de la Compilación de Justiniano fue el limitado conocimiento de la lengua latina, en la parte oriental del Imperio, que hablaba griego ${ }^{49}$. Por tanto, Teófilo, introduciendo a los estudiantes del primer año en los estudios jurídicos, mediante la utilización de las Instituciones de Justiniano como manual que sirviera de base a la aproximación a la materia, escribió, probablemente, la propia Paráfrasis en griego para intentar poner remedio al problema lingüístico. A mi parecer, el profesor no violó la prohibición de Justiniano claramente establecida en la Constitución Tanta-Dédoken del año 533, con la cual fue promulgado el Digesto. En dicha constitución, redactada tanto en latín como en griego, como se ve también en su doble nombre, se afirma, en el parágrafo $21^{50}$, que está prohibido hacer comentarios de estos textos

48 Arangio-Ruiz, Vincenzo-Guarino, Antonio (1983) Breviarium iuris Romani. 6a edición. Milano: Giuffrè Editore, p. 198, nota 3.

49 Falcone, Giuseppe (1998). "Il metodo di compilazione delle Institutiones di Giustiniano". Annali del Seminario Giuridico della Università di Palermo, 45.1, p. 244.

50 Parágrafo 21 de la Constitución Tanta-Dédoken: “... tempestivum nobis videtur et in presenti sancire, ut nemo neque eorum, qui in praesenti iuris peritiam habent, nec qui postea fuerint audeat commentarios isdem legibus adnectere: nisi tantum si velit eas in Graecam vocem transformare sub eodem ordine eaque consequentia, sub qua et voces Romanae positae sunt (hoc quod

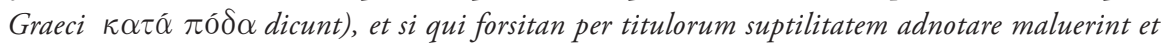
ea quae $\pi \alpha \rho \alpha \tau \iota \tau \lambda \alpha$ nuncupantur componere” (“... nos parece oportuno decretar al presente. .. que nadie de los que en la actualidad poseen el conocimiento del derecho, ni de los que en lo sucesivo lo adquieran, se atreva á agregar comentarios á estas leyes, sino que tan solo pueda, si 
normativos, excepto que si se quiere traducirlos al griego, manteniendo el mismo orden y la misma sucesión en que las palabras latinas están puestas (Katà póda), y también está prohibido añadirle notas (parátitla) y sumar párrafos como integraciones a los títulos. Sin embargo, Justiniano aclara que tal prohibición se ha hecho necesaria después de que el Edicto perpetuo, a causa de los varios comentarios, quedó reducido a la indeterminación ${ }^{51}$. Por lo tanto, si alguien hubiese realizado comentarios a la obra apenas redactada, sufriría la pena por el crimen de falsedad y sus libros serían destruidos ${ }^{52}$.

La violación de la prohibición establecida por Justiniano, como acabamos de ver, no se referiría, según mi opinión, a la Paráfrasis misma que, en la parte que a nosotros interesa, no es otra cosa que una reproposición, con un lenguaje propio, de la actio tributoria de las Instituciones imperiales. Además, sería impensable suponer que Teófilo se haya expuesto intencionalmente a la imputación por un crimen de falsedad con la consiguiente destrucción de la obra.

Algunos, luego, han sostenido que la Paráfrasis sería el conjunto de los apuntes de un estudiante. Esta teoría, criticada por muchos a los cuales yo me asocio, es muy discutible por el solo hecho de que parece extraño que un estudiante de primer año pudiera tener tal conocimiento del derecho y la propiedad de lenguaje en un grado de ecribirla.

\section{7) ANÁlisis de SU CONTENIDO}

Hechas estas precisiones, observemos el párrafo de la Paráfrasis en 4, 7, 3: "El pretor también introdujo otra acción, que llamamos tributoria. De hecho, si mi esclavo ejercita una actividad comercial con su mercancía del peculio, ni habiéndolo ordenado yo ni habiéndolo designado, pero, sin embargo, sabiéndolo y no oponiéndome, y alguna cosa se hubiera contratado con él, así

quisiere, traducirlas á la lengua griega, con el mismo orden y la misma correlación con que han sido escritas en lengua romana (lo que dicen los griegos $\kappa \alpha \tau \alpha ́ \alpha \quad \tau o ́ \delta \alpha$-al pie de la letra-), y componer lo que llaman paratitlos, si por acaso prefiriesen hacer algunas anotaciones por causa de la dificultad de los titulos"). Véanse Mommsen (1928) 22 y García del Corral (2004) 186.

51 Parágrafo 21 de la Constitución Tanta-Dédoken: "Quod et in antiquis edicti perpetui commentatoribus factum est, qui opus moderate confectum huc atque illuc in diversas sententias producentes in infinitum detraxerunt, ut paene omnem Romanam sanctionem esse confusam" ("Cosa que se hizo por los antiguos comentaristas del Edicto perpétuo, quienes extendieron hasta lo infinito una obra confeccionada con moderación, alargándola acá y acullá con diversos pareceres, hasta el punto de haber sido confundida casi toda la legislación romana"). Véanse MommSEN (1928) 22 y García del Corral (2004) 186.

52 Parágrafo 22 de la Constitución Tanta-Dédoken: "Si quid autem tale facere ausi fuerint, ipsi quidem falsitatis rei constituantur, volumina autem eorum omnimodo corrumpentur" ("Mas si tal cosa se hubieren atrevido á hacer, sean considerados como reos de falsedad, y sus libros sean inutilizados por completo"). Véanse Mommsen (1928) 23 y García del Corral (2004) 186. 
de esto enuncia el derecho el pretor. Si se ha obligado a varias personas, entre los cuales también estoy yo que soy el amo (lo que se dice sobre estar obligado no se debe entender como obligación civil, sino natural), y está presente el esclavo que tenía la mercancía peculiar, los acreedores acuden al pretor, el cual constriñe al amo a conferir las mercancias en el activo, y si alguna mercancía fue vendida y de esto hubo recaudación, también se incluye ese dinero, de modo que dicho activo sea distribuido a prorrata entre el amo y todos los demás acreedores. Si en cambio el amo, a quien se permite la distribución de la mercancía, hubiera sustraido alguna cosa de alli o no haya conferido todo en el activo, y se queje alguno de los acreedores y diga que el amo conferió menos mercancias respecto de aquellas con las cuales se ha negociado con el esclavo, le competerá a aquel la acción tributoria contra el amo, con la cual será constreñido a conferir en el activo cualquier mercancía que hubiere escondido. Decimos "tributoria" por distribuir" 53.

De la lectura de la Paráfrasis se contempla nuevamente, in primis, la introducción de la acción tributoria por parte del pretor y el requisito del conocimiento del amo. Como se pone en evidencia, sin embargo, Teófilo es mucho más preciso y detallado respecto de cuanto vimos en Gayo y en las Instituciones de Justiniano, donde se reporta solo el término "sciens", y esto podría ser interpretado precisamente con el espíritu con que se hace una paráfrasis. Aquí, en efecto, se precisa que, además de estar en conocimiento, el amo tampoco debe haber contradicho ni haberla ordenado ni haber designado al esclavo como gerente con las mercancías del peculio. Esto nos podría hacer pensar, queriendo hacer un paralelo con el comentario al Edicto de Ulpiano antes visto, en una tolerancia a la actividad. Teófilo explica que este tipo de obligación debe considerarse natural y no civil, porque como habíamos dicho al inicio hablando en general de las actiones adiecticiae qualitatis, una responsabilidad del dominus por las obligaciones asumidas por el esclavo no estaba prevista en el ius civile, el cual no permitía un empeoramiento de la situación económica del amo a

53 En la traducción latina que se hace en la edición de Ferrini: "Introduxit et aliam actionem praetor, quae tributoria vocatur. Namque si servus meus in peculiaribus suis mercibus negotietur, me non iubente neque praeponente, set tamen sciente et non contradicente, et quid cum eo fuerit contractum, ita de hoc praetor ius dicit. Si pluribus obnoxius factus sit, inter quos et mihi domino (quod autem dixi obnoxium esse de naturali non civili obligatione accipiendum est), et adsit servus merces suas habens, adeunt creditores praetorem, qui coget dominum merces in medium proferre et si qua merx distracta fuerit et inde pecunia recepta, hanc inter dominum et ceteros creditores pro rata portione distribuere. Sin autem dominus, cui mercium distributio est permissa, aliquid inde subtraxerit neque omnia in medium protulerit, et queratur quidam ex creditoribus et dicat pauciores merces, quam in quibus negotiatus sit servus, a domino in medium esse prolatas, tributoria actio ei adversus dominum competet, qua is cogetur quascumque condiderit merces in medium proferre. Dicitur autem 'tributoria' a 'distribuendo"'. 
causa de su propio sometido. Tal precisión no está presente ni en Gayo ni en Justiniano, lo cual nos podría señalar, aun más, el papel formativo de la Paráfrasis.

En la continuación del fragmento, se lee que los acreedores acuden al pretor a fin de que se proceda a la distribución de las mercancías, repartiendo también el precio que se cobró de lo que ha sido vendido. El acudir al pretor para proceder a la distribución, repropone la pregunta de si efectivamente el magistrado hubiera emitido un decretum para dar curso al procedimiento y disciplinarlo. En la doctrina, muchos han pensado que la intervención del pretor, extraída de la Paráfrasis, tendría que identificarse con una evolución bizantina o también que la ausencia en las Instituciones de Gayo se justificaría con un interés metodológico suyo en la descripción de la actio tributoria. Sin embargo, correctamente se afirmó que no se entiende por qué se debe hablar de una modernización del derecho, respecto de la época clásica en la que escribe Gayo, cuando es precisamente gracias al mismo Teófilo que pudimos saber más acerca de la bonorum venditio respecto de lo que es mencionado por las fuentes clásicas ${ }^{54}$. En efecto el Antecessor no solo se formó en base a los textos clásicos, sino también enseñó el derecho a los alumnos mediante el manual institucional de Gayo, empleado hasta la reforma de los estudios jurídicos por parte de Justiniano dispuesta en la Constitución Omnen del 15 de diciembre del 533. En consecuencia, tal interpretación me encuentra del todo favorable porque el intento del profesor de Constantinopla, en esta óptica, era solo el de describir la disciplina de la actio tributoria en la época clásica. Recordemos, además, que el edicto del pretor no contenía órdenes para los ciudadanos, sino que prospectaba un programa mediante promesas de instrumentos judiciales e indicaciones de modelos de remedios que hubiera aprobado. Por lo tanto, tenía la facultad de emitir unos decreta para disciplinar el caso que no hubiese previsto en el edicto.

\section{8) BREVES NOTAS CONCLUSIVAS}

A la luz de lo que acabamos de decir por ende, aun hoy en día el estudio de la actio tributoria ofrece unos puntos de reflexión, que sin ninguna pretención de sostener la existencia de una continuidad histórica linear entre derecho romano y derecho positivo moderno, vale la pena considerar con referencia bien sea a la legislación italiana vigente (Regio decreto 16 de marzo de 1942 n. 267 y sucesivos cambios), así como a la legislación chilena (el 9 de enero de 2014 se publicó en el Diario Oficial

54 Talamanca, Mario (1993-1994). rec. a Chiusi, Tiziana Jolanda (1993), "Contributo allo studio dell'editto "De tributoria actione»". Memorie, Atti della Accademia Nazionale dei Lincei, Serie IX, vol. III, N 4, pp. 277-397, Bullettino dell'Istituto di Diritto Romano, pp. 35-36; 708 . 
la Ley 20.720 entrada en vigencia el 10 de octubre 2014), en materia de procedimientos concursales.

A tal respecto es útil recordar como ambas leyes sean dominadas por el principio de la par condicio creditorum (igual posición de los acreedores) que representa la verdadera esencia del procedimiento concursal de la llamada a la contribución (vocare in tributum) y de la acción tributoria. Por consecuente -es oportuno subrayarlo una vez más- parece necesario corregir la opinión común según la cual dicho fundamento del derecho concursal moderno nacería solo en los Estatutos de las ciudades mercantiles italianas en la baja Media Edad, porque en cambio ya era conocido en la jurisdicción del pretor romano. Además si se agrega que en las fuentes jurídicas, parece existir una cierta terminología específica para algunos casos de insolvencia que se refieren únicamente a un negotiator ${ }^{55}$, los puntos de reflexión sobre este tema, en el marco del derecho romano, demuestran la oportunidad de una investigación mucho más completa y profunda.

\section{BIBLIOGRAFÍA CITADA}

Andreau, Jean (1968) "Banque grecque et banque romaine dans le théâtre de Plaute et de Térence". Mélanges de l'École Française de Rome, vol.

Arangio-Ruiz, Vincenzo-Guarino, Antonio (1983). Breviarium iuris Romani. 6a edición. Milano: Giuffrè Editore.

Balestri Fumagalli, Marcella (1988) "L'«actio tributoria» nel sistema delle opere istituzionali di Gaio, di Giustiniano e di Teofilo". VV.AA. Atti del seminario sulla problematica contrattuale in diritto romano, Milano, 7-9 aprile 1987. Milano: Cisalpino-Goliardica, I.

Buckland, William Warwick (1908) The Roman law of slavery. The condition of the slave in private law from Augustus to Justinian. Cambridge: at the University Press.

Burdese, Alberto (2010) Manuale di diritto privato romano. Rist. Milano: Wolters Kluwer Italia.

Cassarino, Alessandro (2014) "Riflessioni sulla nozione di 'Foro cedere' in riferimento all' esecutato nel diritto romano tra tarda repubblica e principato". Teoria e Storia del Diritto Privato, 7, 1.

Cerami, Pietro (2010) "Introduzione allo studio del diritto commerciale romano”. En Cerami, Pietro-Petrucci, Aldo: Diritto commerciale romano. Profilo storico. 3a edición. Torino: Giappichelli Editore.

\footnotetext{
55 Al respecto me permito indicar mi estudio CASSARIno, Alessandro (2014). "Riflessioni sulla nozione di 'Foro cedere' in riferimento all'esecutato nel diritto romano tra tarda repubblica e principato". Teoria e Storia del Diritto Privato, 7, pp. 1 y ss.
} 
Chiusi, Tiziana Jolanda (1993) "Contributo allo studio dell'editto «De tributoria actione»". Memorie, Atti della Accademia Nazionale dei Lincei, Serie IX, Vol. III, N4.

De Ligt, Luuk (1999) "Legal history and economic history: the case of the actiones adiecticiae qualitatis". Tijdschrift voor Rechtsgeschiedenis, 67.

FAlCone, Giuseppe (1998) "Il metodo di compilazione delle Institutiones di Giustiniano". Annali del Seminario Giuridico della Università di Palermo, 45.1.

Fleckner, Andreas Michael (2014) “The Peculium: A Legal Device for Donations to personae alieno iuri subiectae?". Gift Giving and the 'Embedded' Economy in the Ancient Worl, Heidelberg: Universitätsverlag Winter.

Földi, András (1995) "Die Entwicklung der sich auf die Schiffer beziehenden Terminologie im römischen Recht". Tijdschrift voor Rechtsgeschiedenis, 63.

FöLDI, András (1996) "Remarks on the legal structure of enterprises in Roman Law". Revue Internationale de Droits de l'Antiquité, 43.

García del Corral, Ildefonso Luis (1889) Cuerpo del Derecho Civil Romano. Barcelona: Jaime Molinas, Editor.

Guzmán Brito, Alejandro (2013) Derecho privado romano. 2a edición. Santiago de Chile: Editorial Juridica de Chile, II.

Lazo, Patricio (2015) "Acciones adyecticias y limitación de responsabilidad. Una hipótesis en torno a la justicia y la utilidad en el pensamiento de Ulpiano". Revista de Estudios Histórico-Jurídicos, vol. 37.

Lenel, Otto (1927) Das Edictum Perpetuum. Leipzig: Verlag von Bernhard Tauchnitz.

Mantovani, Dario (1999) Le formule del processo privato romano. Per la didattica delle Istituzioni di diritto romano. Padova: Milano.

Marrone, Matteo (2006) Istituzioni di diritto romano. Palermo: Palumbo \& C. Editore.

MiCELI, Maria (2001) Sulla struttura formulare delle 'actiones adiecticiae qualitatis'. Torino: Giappichelli Editore.

Petrucci, Aldo (1991) Mensam exercere. Studi sull'impresa finanziaria romana (II secolo a.C.-metà del III secolo d.C.). Napoli: Jovene Editore.

Petrucci, Aldo (2007) Per una storia della protezione dei contraenti con gli imprenditori. Torino: Giappichelli Editore, I.

Petrucci, Aldo (2012) Corso di diritto pubblico romano. Torino: Giappichelli Editore. 
Petrucci, Aldo (2012) “Idee 'vecchie' e 'nuove' sulle attività imprenditoriali gestiti all'interno di un peculio". Bullettino dell'Istituto di Diritto Romano, 106, vol. 2.

Petrucci, Aldo (2014) Nozioni elementari di diritto romano. Pisa: Arnus University Books.

SerraO, Feliciano (1971) "Institore (storia)". Enciclopedia del Diritto. Milano: Giuffrè Editore, vol. 21.

Serrao, Feliciano (2002) Impresa e responsabilità a Roma nell'età commerciale. Forme giuridiche di un'economia-mondo. Pisa: Pacini Editore.

Serrao, Feliciano (2006) Diritto privato economia e società nella storia di Roma. Dalla società gentilizia alle origini dell'economia schiavistica. Napoli: Jovene Editore, I.

SuÁrez BlázQuez, Guillermo (2013) "Concurso mercantil de acreedores: vocatio in tributum. La "Acción tributoria" frente al dueño (empresario corrupto)". Revista de Derecho, Universidad del Norte, 39.

Talamanca, Mario (1990) Istituzioni di diritto romano. Milano: Giuffrè.

Talamanca, Mario (1993-1994) rec. a Chiusi, Tiziana Jolanda (1993), "Contributo allo studio dell'editto "De tributoria actione"". Memorie, Atti della Accademia Nazionale dei Lincei, Serie IX, vol. III, $\mathrm{N}^{\circ} 4$.

VAliño, Emilio (1967) "La 'actio tributoria". Studia et Documenta Historiae et Iuris, vol. 33. 